\title{
Letter
}

\section{On the triplet anti-triplet symmetry in 3-3-1 models}

\author{
Le Tho Hue ${ }^{1,2}$, Le Duc Ninh ${ }^{3, a}$ \\ ${ }^{1}$ Institute for Research and Development, Duy Tan University, Da Nang 550000, Vietnam \\ ${ }^{2}$ Institute of Physics, Vietnam Academy of Science and Technology, 10 Dao Tan, Ba Dinh, Hanoi, Vietnam \\ ${ }^{3}$ Institute For Interdisciplinary Research in Science and Education, ICISE, Quy Nhon 590000, Vietnam
}

Received: 26 December 2018 / Accepted: 28 February 2019 / Published online: 11 March 2019

(C) The Author(s) 2019

\begin{abstract}
We present a detailed discussion of the triplet anti-triplet symmetry in 3-3-1 models. The full set of conditions to realize this symmetry is provided, which includes in particular the requirement that the two vacuum expectation values of the two scalar triplets responsible for making the $W$ and $Z$ bosons massive must be interchanged. We apply this new understanding to the calculation of processes that have a $Z-Z^{\prime}$ mixing.
\end{abstract}

\section{Introduction}

Interesting extensions of the Standard Model (SM), based on the local gauge group $S U(3)_{C} \otimes S U(3)_{L} \otimes U(1)_{X}$ (3-3$1)$, have been widely studied (see Ref. [1] and references therein, see also Refs. [2-6] for similar models but with lepton-number violation). Fermions are typically organized into triplets and anti-triplets of $S U(3)_{L}$ in three generations. We therefore have two possible choices, either to put leptons in triplets or anti-triplets.

The 3-3-1 models can be classified using the parameter $\beta$ defined via the electric charge operator

$Q=T_{3}+\beta T_{8}+X \mathbb{1}$,

where $T_{3}, T_{8}$ are the diagonal $S U(3)$ generators and $X$ is a new quantum charge of the group $U(1)_{X}$.

For a given $\beta \neq 0$, the model with the leptons in triplets is different from the one where the leptons are in anti-triplets, because the two models have different electric-charge spectra. Also, for a given assignment of fermionic representation, changing the sign of $\beta$ will lead to a different electric-charge spectrum. What happens if we switch both simultaneously? We call this

$\beta \rightarrow-\beta$ and triplets $\leftrightarrow$ anti-triplets,

a e-mail: 1dninh@ifirse.icise.vn triplet anti-triplet transformation for short. This transformation applies also to quarks, i.e. quark triplets $\rightarrow$ quark antitriplets and quark anti-triplets $\rightarrow$ quark triplets. In the special case of $\beta=0$ [7], this becomes truly a triplet anti-triplet transformation literally.

Using Eq. (1) it is possible to show that the electric-charge spectrum is invariant. Therefore, at first glance, we expect that physics must be the same. This was noted e.g. in Ref. [8] (see the sentence after Eq. (2.4) therein) and at the end of Section II in Ref. [9]. We think that this is well recognized in the 3-3-1 model community.

However, this understanding is put into question, at least for us, after reading the paper of Buras, De Fazio and Girrbach-Noe [10] (see also [11]) where there are indications that this symmetry is broken by $Z-Z^{\prime}$ mixing, which depends on the sign of $\beta$ but, apparently, not on the fermionic representation at tree level. More specifically, the $Z-Z^{\prime}$ mixing angle is given, in $M_{Z} \ll M_{Z^{\prime}}$ approximation, by [10]

$\sin \xi=\frac{c_{W}^{2}}{3 \sqrt{1-\left(1+\beta^{2}\right) s_{W}^{2}}}\left(3 \beta \frac{s_{W}^{2}}{c_{W}^{2}}+\sqrt{3} a\right) \frac{M_{Z}^{2}}{M_{Z^{\prime}}^{2}}$,

where $s_{W}^{2}=\sin ^{2} \theta_{W}, c_{W}^{2}=1-s_{W}^{2}$ with $\theta_{W}$ being the weakmixing angle and

$a=\frac{v_{1}^{2}-v_{2}^{2}}{v_{1}^{2}+v_{2}^{2}}$

where $v_{1}$ and $v_{2}$ (called $v_{\eta}$ and $v_{\rho}$ in Ref. [10], respectively) are the vacuum expectation values of the two Higgs triplets responsible for making $W^{ \pm}$and $Z$ bosons massive. From Eq. (3), one can see that the absolute value of the mixing angle changes under $\beta \rightarrow-\beta$. The authors of Ref. [10] further pointed out that $\sin \xi$ does not depend on whether the leptons are assigned in triplets or anti-triplets. This seems to indicate that the $Z-Z^{\prime}$ mixing breaks the triplet antitriplet symmetry, see the discussion around Eq. (2.16) of 
Ref. [10]. Many figures in Ref. [10] also seem to support this conclusion.

We notice, however, one missing ingredient in the above discussion. Namely, the parameter $a$ defined in Eq. (4) changes sign under the triplet anti-triplet transformation. The authors of Ref. [10] did not see this because they chose $a$ to be an input parameter and kept it unchanged under the transformation. If we instead choose the charged gauge-boson masses as independent input parameters and calculate $a$ from them, then we will see that the value of $a$ changes sign. This is the main point of this letter, which, to the best of our knowledge, has not been noted in the literature. The choice of the charged-gauge boson masses as input parameters is natural as this is directly related to physical observables. Reference [10] focused on the neutral gauge bosons and did not touch the charged gauge-boson masses, hence this important point was missed out. With this new piece of information, we will see that $\sin \xi$ can only change sign under the triplet anti-triplet transformation.

In trying to solve this puzzle, we have realized that there exists no detailed discussion of the triplet anti-triplet symmetry in the literature apart from some brief remarks as above noted. Since this is an important issue in 3-3-1 models, we think it can be useful to show in detail how this symmetry works. We have found that the actual implementation of this symmetry in practice requires not only a careful attention to the input parameter scheme as above noted but also possible sign flips in many places in the Feynman rules and in bookkeeping parameters. We will also show that the full definition of the triplet anti-triplet transformation is more complicated than Eq. (2) and changing the sign of the parameter $a$. This is easy to see because the full Lagrangian depends also on many other parameters, which may also flip signs or interchange under the transformation.

There is another issue related to the comparison with Ref. [10]. Indeed, Ref. [10] provided results for two models called $F_{1}(\beta, a)$ and $F_{2}(-\beta, a)$, related by the transformation Eq. (2). For each model, results for different values $a=0, \pm 12 / 13$ are also given. Numerical results of Ref. [10], see Fig. 4 and Fig. 5 therein, show that $F_{1}(\beta, a)$ and $F_{2}(-\beta,-a)$ are not the same. This is very surprising to us because we expect them to be identical according to the triplet anti-triplet symmetry. We have discussed this issue with the authors of Ref. [10], but, unfortunately, no conclusive finding has been reached. Our investigation has led us to the conclusion that there seems to be an issue with the sign of the couplings between the $Z^{\prime}$ and the leptons in the model $F_{2}$.

The paper is organized as follows. In Sect. 2, we discuss the two models related by the transformation Eq. (2) and provide the full set of conditions for them to be identical. In Sect. 3, we make application to the processes with a $Z-Z^{\prime}$ mixing and perform some crosschecks with Ref. [10] and other papers. Conclusions are given in Sect. 4. In Appendix A we provide details on the calculation of the $Z-Z^{\prime}$ mixing and of the couplings between the $Z, Z^{\prime}$ gauge bosons to the leptons in the two models with a general sign convention for the $Z^{\prime}$ field definition.

\section{Two identical models}

In this section we consider two 3-3-1 models denoted $M_{1}$ and $M_{2}$, related by the triplet anti-triplet transformation defined by Eq. (2). We note that Eq. (2) is not enough to make the two models identical, because the physical results depend also on the values of other input parameters such as masses, mixing and coupling parameters. Since we impose here that the two models are identical, there must be relations between the parameters of the two models. These relations can be found by comparing the two Lagrangians.

The parameter $\beta$ will be denoted $\beta_{1}$ and $\beta_{2}$ for the two models, respectively. We will use the indices $m, n=1,2$ to distinguish the models.

The model $M_{1}$ is defined as follows. Left-handed leptons are assigned into anti-triplets and right-handed leptons are singlets:

$$
\begin{aligned}
L_{a L} & =\left(\begin{array}{c}
e_{a} \\
-v_{a} \\
E_{a}
\end{array}\right)_{L} \sim\left(3^{*},-\frac{1}{2}+\frac{\beta_{1}}{2 \sqrt{3}}\right), \quad a=1,2,3, \\
e_{a R} & \sim(1,-1), \quad v_{a R} \sim(1,0) \\
E_{a R} & \sim\left(1,-\frac{1}{2}+\frac{\sqrt{3} \beta_{1}}{2}\right) .
\end{aligned}
$$

The model includes three right-handed neutrinos $v_{a R}$. This is to make the lepton sector similar to the quark one, in particular the Yukawa Lagrangian. Discussions and results for the leptons are then easier to be adopted for the quarks. For the same reason, possible Majorana mass terms have been neglected. The leptons $E_{L, R}^{a}$ can be new particles or chargeconjugated states of the SM leptons. In the following, we will assume, without loss of generality, $E_{a}$ to be new leptons. The numbers in the parentheses are to label the representations of $S U(3)_{L}$ and $U(1)_{X}$ groups. Note that we have $Q=X$ for singlets.

Anomaly cancellation requires that the number of triplets and anti-triplets must be equal. Since quarks come in three colors, this means that one family of quarks must be in antitriplet and the other two families are in triplets or vice versa. This implies two choices, the leptons are either put in triplets or in anti-triplets. Because Feynman rules for the quarks are similar to those for the leptons, we will ignore the quarks and focus on the leptons in the following. 
For $M_{2}$, the left-handed leptons are put in triplets as

$$
\begin{aligned}
L_{a L} & =\left(\begin{array}{c}
v_{a} \\
e_{a} \\
E_{a}
\end{array}\right)_{L} \sim\left(3,-\frac{1}{2}-\frac{\beta_{2}}{2 \sqrt{3}}\right), \quad a=1,2,3, \\
e_{a R} & \sim(1,-1), \quad v_{a R} \sim(1,0), \\
E_{a R} & \sim\left(1,-\frac{1}{2}-\frac{\sqrt{3} \beta_{2}}{2}\right) .
\end{aligned}
$$

Note that the positions of $v_{a L}$ and $e_{a L}$ have been interchanged to make the Feynman rules for the SM particles the same as those in the SM. Requiring that the electric charges of $E_{a}$ in both models are the same leads to

$\beta_{1}=-\beta_{2}$

We use the same convention for the Lagrangian of both models as

$$
\begin{aligned}
\mathcal{L}_{\text {lepton }}= & \bar{L}_{a L} i \gamma^{\mu} D_{\mu} L_{a L}+\bar{e}_{a R} i \gamma^{\mu} D_{\mu} e_{a R} \\
& +\bar{v}_{a R} i \gamma^{\mu} D_{\mu} v_{a R}, \\
\mathcal{L}_{\text {scalar }}^{\text {kinetic }}= & \left(D_{\mu} \Phi_{i}\right)^{\dagger}\left(D^{\mu} \Phi_{i}\right) \\
\mathcal{L}_{1, \text { Yukawa }}= & -Y_{1, a b}^{e} \bar{L}_{a L} \Phi_{1}^{*} e_{b R} \\
& -Y_{1, a b}^{v} \bar{L}_{a L} \Phi_{2}^{*} v_{b R}-Y_{1, a b}^{E} \bar{L}_{a L} \Phi_{3}^{*} E_{b R}+\text { h.c. },
\end{aligned}
$$

$$
\begin{aligned}
\mathcal{L}_{2, \text { Yukawa }}= & -Y_{2, a b}^{e} \bar{L}_{a L} \Phi_{2}^{*} e_{b R}-Y_{2, a b}^{v} \bar{L}_{a L} \Phi_{1}^{*} v_{b R} \\
& -Y_{2, a b}^{E} \bar{L}_{a L} \Phi_{3}^{*} E_{b R}+\text { h.c. },
\end{aligned}
$$

where $i=1,2,3$ to denote the three scalar multiplets. To give mass to the new particles, to all the SM leptons, and to the up- and down-type quarks at tree level, we need at least three scalar multiplets and use two of them to break the $S U(2)_{L} \otimes$ $U(1)_{Y}$ symmetry, with $Y$ being the usual hypercharge. Other reduced scalar sector choices such as using two scalar (anti)triplets, apart from possible problems with giving mass to the quarks, may force the scalar potential to be in a particular form which can be strongly distorted by radiative corrections. This may lead to difficulties in the renormalization step. The Yukawa Lagrangians are written for both models explicitly. The Yukawa couplings are the same for both models, namely

$Y_{1, a b}^{l}=Y_{2, a b}^{l}, \quad l=e, v, E$.

The covariant derivative reads

$$
\begin{aligned}
D_{\mu}^{\text {triplet }} & \equiv \partial_{\mu}-i g T_{s} W_{\mu}^{s}-i g_{X} X T_{9} X_{\mu}, \\
D_{\mu}^{\text {anti-triplet }} & \equiv \partial_{\mu}+i g\left(T_{s}\right)^{T} W_{\mu}^{s}-i g_{X} X T_{9} X_{\mu}, \\
D_{\mu}^{\text {singlet }} & \equiv \partial_{\mu}-i g_{X} X T_{9} X_{\mu},
\end{aligned}
$$

where $T_{s}=\lambda_{s} / 2$ with $s=1, \ldots, 8$ and $\lambda_{s}$ being Gell-Mann matrices, $T_{9}=1 / \sqrt{6}, g$ and $g_{X}$ are coupling constants corre- sponding to the two groups $S U(3)_{L}$ and $U(1)_{X}$, respectively. Their values are the same in both models. We further define

$$
\begin{aligned}
\mathcal{W}_{\mu}^{\text {triplet }} & \equiv W_{\mu}^{s} T_{s} \\
& =\frac{1}{2}\left(\begin{array}{ccc}
W_{\mu}^{3}+\frac{1}{\sqrt{3}} W_{\mu}^{8} & \sqrt{2} W_{\mu}^{+} & \sqrt{2} Y_{\mu}^{+A_{m}} \\
\sqrt{2} W_{\mu}^{-} & -W_{\mu}^{3}+\frac{1}{\sqrt{3}} W_{\mu}^{8} & \sqrt{2} V_{\mu}^{+B_{m}} \\
\sqrt{2} Y_{\mu}^{-A_{m}} & \sqrt{2} V_{\mu}^{-B_{m}} & -\frac{2}{\sqrt{3}} W_{\mu}^{8}
\end{array}\right), \\
\mathcal{W}_{\mu}^{\text {anti-triplet }} & \equiv-W_{\mu}^{s}\left(T_{s}\right)^{T}=-\left(\mathcal{W}_{\mu}^{\text {triplet }}\right)^{T},
\end{aligned}
$$

where $m=1,2$ and we have defined the mass eigenstates of the charged gauge bosons as

$$
\begin{aligned}
W_{\mu}^{ \pm} & =\frac{1}{\sqrt{2}}\left(W_{\mu}^{1} \mp i W_{\mu}^{2}\right), \\
Y_{\mu}^{ \pm A_{m}} & =\frac{1}{\sqrt{2}}\left(W_{\mu}^{4} \mp i W_{\mu}^{5}\right), \\
V_{\mu}^{ \pm B_{m}} & =\frac{1}{\sqrt{2}}\left(W_{\mu}^{6} \mp i W_{\mu}^{7}\right) .
\end{aligned}
$$

The electric charges of the gauge bosons are calculated as

$$
A_{m}=\frac{1}{2}+\beta_{m} \frac{\sqrt{3}}{2}, \quad B_{m}=-\frac{1}{2}+\beta_{m} \frac{\sqrt{3}}{2} .
$$

We see clearly that $\beta_{2}=-\beta_{1}$ is equivalent to $B_{m}=-A_{n}$ with $m \neq n$.

Equations (10) and (11) require that $\Phi_{i}$ are triplets in $M_{1}$ and anti-triplets in $M_{2}$. This is just a matter of convention and we can e.g. change $\Phi_{i}$ to be triplets in $M_{2}$ by removing the complex conjugation in Eq. (11). For $M_{1}$ we have

$$
\begin{aligned}
& \Phi_{3}=\left(\begin{array}{c}
\phi_{3}^{+A_{1}} \\
\phi_{3}^{+B_{1}} \\
\phi_{3}^{0}
\end{array}\right) \sim\left(3, \frac{\beta_{1}}{\sqrt{3}}\right), \\
& \Phi_{2}=\left(\begin{array}{c}
\phi_{2}^{+} \\
\phi_{2}^{0} \\
\phi_{2}^{-B_{1}}
\end{array}\right) \sim\left(3, \frac{1}{2}-\frac{\beta_{1}}{2 \sqrt{3}}\right) \\
& \Phi_{1}=\left(\begin{array}{c}
\phi_{1}^{0} \\
\phi_{1}^{-} \\
\phi_{1}^{-A_{1}}
\end{array}\right) \sim\left(3,-\frac{1}{2}-\frac{\beta_{1}}{2 \sqrt{3}}\right) .
\end{aligned}
$$

And for $M_{2}$

$$
\begin{aligned}
& \Phi_{3}=\left(\begin{array}{c}
\phi_{3}^{-A_{2}} \\
\phi_{3}^{-B_{2}} \\
\phi_{3}^{0}
\end{array}\right) \sim\left(3^{*}, \frac{-\beta_{2}}{\sqrt{3}}\right), \\
& \Phi_{2}=\left(\begin{array}{c}
\phi_{2}^{-} \\
\phi_{2}^{0} \\
\phi_{2}^{+B_{2}}
\end{array}\right) \sim\left(3^{*},-\frac{1}{2}+\frac{\beta_{2}}{2 \sqrt{3}}\right)
\end{aligned}
$$




$$
\Phi_{1}=\left(\begin{array}{c}
\phi_{1}^{0} \\
\phi_{1}^{+} \\
\phi_{1}^{+A_{2}}
\end{array}\right) \sim\left(3^{*}, \frac{1}{2}+\frac{\beta_{2}}{2 \sqrt{3}}\right) .
$$

The scalar fields develop vacuum expectation values (VEV) defined as

$$
\begin{aligned}
& \left\langle\Phi_{3}\right\rangle=\frac{1}{\sqrt{2}}\left(\begin{array}{c}
0 \\
0 \\
v_{m, 3}
\end{array}\right), \\
& \left\langle\Phi_{2}\right\rangle=\frac{1}{\sqrt{2}}\left(\begin{array}{c}
0 \\
v_{m, 2} \\
0
\end{array}\right), \quad\left\langle\Phi_{1}\right\rangle=\frac{1}{\sqrt{2}}\left(\begin{array}{c}
v_{m, 1} \\
0 \\
0
\end{array}\right) .
\end{aligned}
$$

We now discuss gauge boson masses. From Eq. (9) we get for charged gauge bosons

$m_{W}^{2}=\frac{g^{2}}{4}\left(v_{m, 1}^{2}+v_{m, 2}^{2}\right)$,

$m_{Y^{ \pm A} A_{m}}^{2}=\frac{g^{2}}{4}\left(v_{m, 3}^{2}+v_{m, 1}^{2}\right)$,

$m_{V^{ \pm B_{m}}}^{2}=\frac{g^{2}}{4}\left(v_{m, 3}^{2}+v_{m, 2}^{2}\right)$.

As noticed, under the transformation $\beta_{2}=-\beta_{1}$ we have $A_{m}=-B_{n}$ with $m \neq n$, hence these equations lead to

$v_{1,3}=v_{2,3}, \quad v_{m, 1}=v_{n, 2}$,

which come from the condition that both models must have the same charged gauge bosons (i.e. same electric charges and same masses). It is straight forward to see that the neutral gauge bosons have the same masses in both models, see "Appendix A". It is convenient to introduce the following parameter

$a_{m}=\frac{v_{m, 1}^{2}-v_{m, 2}^{2}}{v_{m, 1}^{2}+v_{m, 2}^{2}}$,

which was defined in Ref. [10] and was mentioned in the introduction. Because of Eq. (21), we have

$a_{1}=-a_{2}$.

We now consider the scalar potentials, which read

$$
\begin{aligned}
V_{m}= & \mu_{m, 1}^{2} \Phi_{1}^{\dagger} \Phi_{1}+\mu_{m, 2}^{2} \Phi_{2}^{\dagger} \Phi_{2}+\mu_{m, 3}^{2} \Phi_{3}^{\dagger} \Phi_{3} \\
& +\lambda_{m, 1}\left(\Phi_{1}^{\dagger} \Phi_{1}\right)^{2} \\
& +\lambda_{m, 2}\left(\Phi_{2}^{\dagger} \Phi_{2}\right)^{2}+\lambda_{m, 3}\left(\Phi_{3}^{\dagger} \Phi_{3}\right)^{2} \\
& +\lambda_{m, 12}\left(\Phi_{1}^{\dagger} \Phi_{1}\right)\left(\Phi_{2}^{\dagger} \Phi_{2}\right)+\lambda_{m, 13}\left(\Phi_{1}^{\dagger} \Phi_{1}\right)\left(\Phi_{3}^{\dagger} \Phi_{3}\right) \\
& +\lambda_{m, 23}\left(\Phi_{2}^{\dagger} \Phi_{2}\right)\left(\Phi_{3}^{\dagger} \Phi_{3}\right) \\
& +\tilde{\lambda}_{m, 12}\left(\Phi_{1}^{\dagger} \Phi_{2}\right)\left(\Phi_{2}^{\dagger} \Phi_{1}\right) \\
& +\tilde{\lambda}_{m, 13}\left(\Phi_{1}^{\dagger} \Phi_{3}\right)\left(\Phi_{3}^{\dagger} \Phi_{1}\right)+\tilde{\lambda}_{m, 23}\left(\Phi_{2}^{\dagger} \Phi_{3}\right)\left(\Phi_{3}^{\dagger} \Phi_{2}\right) \\
& +\sqrt{2} f_{m}\left(\epsilon_{i j k} \Phi_{1}^{i} \Phi_{2}^{j} \Phi_{3}^{k}+\text { h.c. }\right), \quad m=1,2 .
\end{aligned}
$$

In order to have the relations in Eq. (21) we must have, with $m \neq n$,

$\mu_{m, 1}^{2}=\mu_{n, 2}^{2}, \quad \mu_{1,3}^{2}=\mu_{2,3}^{2}, \quad \lambda_{m, 1}=\lambda_{n, 2}, \quad \lambda_{1,3}=\lambda_{2,3}$,

$\lambda_{1,12}=\lambda_{2,12}, \quad \lambda_{m, 13}=\lambda_{n, 23}, \quad \tilde{\lambda}_{1,12}=\tilde{\lambda}_{2,12}$,

$\tilde{\lambda}_{m, 13}=\tilde{\lambda}_{n, 23}, \quad f_{1}=f_{2}$.

With these relations it is straight forward to see that the Higgs mass spectra of the two models are identical and the vertices of pure scalar, scalar-fermion, scalar gauge boson interactions are the same.

In summary, the two models $M_{1}$, where the leptons are organized in anti-triplets, and $M_{2}$, where the leptons are in triplets, are equivalent if, besides identical gauge couplings, the relations Eqs. (7), (12), and (25) are satisfied. The important relation Eq. (23) is a consequence of Eq. (25). We therefore remark that the conditions in Eq. (2) are necessary but not sufficient to realize the triplet anti-triplet symmetry.

\section{Application to neutral-current processes}

For the following discussion, it is useful to define the models as follows

$M_{1}=M\left(3^{*}, \beta_{1}, a_{1}\right), \quad M_{2}=M\left(3, \beta_{2}, a_{2}\right)$,

where the first argument specifies the representation for the leptons. Of course, those three arguments are not enough to define a model, but they will be enough for our purpose in this section, assuming that the gauge and Yukawa couplings are the same, and the parameter $a$ represents the parameters of the scalar potential. With these assumptions, we have

$M\left(3^{*}, \beta, a\right)=M(3,-\beta,-a)$,

as a simplified way of expressing the triplet anti-triplet symmetry.

In Ref. [10], two models are discussed $F_{1}(a)=M\left(3^{*}\right.$, $\beta, a)$ and $F_{2}(a)=M(3,-\beta, a)$. Ref. [10] introduced also the parameter $\tan \bar{\beta}=v_{m, 2} / v_{m, 1}$, which can be related to $a$ via

$a=\frac{1-\tan ^{2} \bar{\beta}}{1+\tan ^{2} \bar{\beta}}$.

The transformation $a \rightarrow-a$ is therefore equivalent to $\tan \bar{\beta} \rightarrow 1 / \tan \bar{\beta}$.

From the above discussion, we can now see clearly that $F_{1}(a)$ and $F_{2}(a)$ are not equivalent, leading (unsurprisingly) to the fact that the results for $F_{1}(a)$ and for $F_{2}(a)$ are not the same if $a \neq 0$.

However, the results of model $F_{2}(-a)$ are also provided in Ref. [10] and they are not the same as those of $F_{1}(a)$. 
This is unexpected because the triplet anti-triplet symmetry suggests that they should be the identical. The calculation of Ref. [10] involves two neutral currents mediated by $Z_{1}$ and $Z_{2}$ particles. These mass eigenstates are related to the $Z$ and $Z^{\prime}$ states as

$Z_{1}^{\mu}=\cos \xi Z^{\mu}+\sin \xi Z^{\prime \mu}, \quad Z_{2}^{\mu}=-\sin \xi Z^{\mu}+\cos \xi Z^{\prime \mu}$.

More details are provided in "Appendix A". The amplitude squared therefore depends on the sign of the $f f Z$ and $f f Z^{\prime}$ couplings and also on the sign of $\tan \xi$, because of the $Z-$ $Z^{\prime}$ interference terms. Since the convention of $\cos \xi>0$ is usually chosen, we thus have to pay attention to the sign of the couplings and of $\sin \xi$.

We have made an investigation into Ref. [10] and come to the conclusion that there seems to be a sign issue in the $l l Z^{\prime}$ couplings of $F_{2}$. We have performed the following checks.

- For model $F_{1}$, we agree with Table 1 of Ref. [10].

- For model $F_{2}$, we agree with Ref. [12].

- For model $F_{2}$, we agree with Ref. [10] on $\sin \xi$ and can reproduce the Table 2 of Ref. [10] if a minus sign is added to the $l l Z^{\prime}$ couplings. ${ }^{1}$ However, if the correct sign is used, the results change because of the $Z-Z^{\prime}$ interference terms.

Note that the sign of $\sin \xi$ in Ref. [12] agrees with Ref. $[10]$ and also with this paper. The $l l Z, l l Z^{\prime}$ couplings of Ref. [12] are the same as in Ref. [13] and agree with this paper. Ref. [10] and Ref. [12] did not mention whether they agree on the $l l Z, l l Z^{\prime}$ couplings.

To facilitate comparisons, our results for the $l l Z, l l Z^{\prime}$ couplings and for $\sin \xi$ are provided in Appendix A. All these findings have been communicated to the authors of Ref. [10].

\section{Conclusions}

In this work we have pointed out that the recognized triplet anti-triplet symmetry in 3-3-1 models should include a sign change in the parameter $a=\left(v_{1}^{2}-v_{2}^{2}\right) /\left(v_{1}^{2}+v_{2}^{2}\right)$, besides the well-known sign change in the parameter $\beta$ and changing from triplets to anti-triplets and vice versa. We have shown that the full transformation is more complicated than that and attention has to be paid to the input parameter

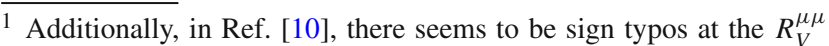
result for $\beta=2 / \sqrt{3}$ and $\tan \bar{\beta}=5$ in Table 1 , and at the $W_{A}^{\mu \mu}$ result for $\beta=-2 / \sqrt{3}$ and $\tan \bar{\beta}=5$ in Table 2 .
}

scheme and also to the parameters of the scalar potential. The transformations of those parameters have been provided.

We have applied the new understanding to the processes with a $Z-Z^{\prime}$ mixing and in particular to the calculations of Ref. [10]. We have found a possible sign issue with the couplings between the $Z^{\prime}$ and the leptons in the model where the leptons are put in the triplet representation.

Acknowledgements We would like to thank Andrzej Buras and Fulvia De Fazio for discussions. We are grateful to Julien Baglio for his careful reading of the manuscript and for his helpful comments. LTH acknowledges the financial support of the International Centre of Physics at the Institute of Physics, Vietnam Academy of Science and Technology. This research is funded by the Vietnam National Foundation for Science and Technology Development (NAFOSTED) under Grant number 103.012017.78. LDN acknowledges the support from DAAD to perform the final part of this work at the University of Tübingen under a scholarship. He thanks the members of the Institut für Theoretische Physik for their hospitality.

Data Availability Statement This manuscript has no associated data or the data will not be deposited. [Authors' comment: This is a theoretical work which does not generate any data sets.]

Open Access This article is distributed under the terms of the Creative Commons Attribution 4.0 International License (http://creativecomm ons.org/licenses/by/4.0/), which permits unrestricted use, distribution, and reproduction in any medium, provided you give appropriate credit to the original author(s) and the source, provide a link to the Creative Commons license, and indicate if changes were made.

Funded by SCOAP ${ }^{3}$.

\section{Appendix A: $Z-Z^{\prime}$ mixing}

We consider here the neutral gauge bosons. For both models defined in Sect. 2, we will introduce intermediate fields of $Z_{\mu}$ and $Z_{\mu}^{\prime}$ and the final physical fields will be $A^{\mu}$ (the photon), $Z_{1}^{\mu}$ and $Z_{2}^{\mu}$. We will see that the masses of these physical states are the same in both models.

The symmetry breaking pattern is

$$
S U(3)_{L} \otimes U(1)_{X} \stackrel{v_{m, 3}}{\longrightarrow} S U(2)_{L} \otimes U(1)_{Y} \stackrel{v_{m, 1}, v_{m, 2}}{\longrightarrow} U(1)_{Q}
$$

The basis of the neutral gauge bosons transforms correspondingly as

$$
X_{\mu}, W_{\mu}^{8}, W_{\mu}^{3} \stackrel{\theta_{m, 331}}{\longrightarrow} Z_{\mu}^{\prime}, B_{\mu}^{\prime}, W_{\mu}^{3} \stackrel{\theta_{W}}{\longrightarrow} Z_{\mu}^{\prime}, Z_{\mu}, A_{\mu} .
$$

We have

$$
\begin{aligned}
A_{\mu} & =s_{W} W_{\mu}^{3}+c_{W}\left(s_{m, 331} X_{\mu}+c_{m, 331} W_{\mu}^{8}\right), \\
Z_{\mu} & =c_{W} W_{\mu}^{3}-s_{W}\left(s_{m, 331} X_{\mu}+c_{m, 331} W_{\mu}^{8}\right), \\
Z_{\mu}^{\prime} & =h_{m}\left(-s_{m, 331} W_{\mu}^{8}+c_{m, 331} X_{\mu}\right),
\end{aligned}
$$


with $h_{m}= \pm 1$ being a sign convention for $Z_{\mu}^{\prime}$, and

$$
\begin{aligned}
& s_{m, 331}=\sin \theta_{m, 331}=\frac{g}{\sqrt{X_{g}}}, \\
& c_{m, 331}=\cos \theta_{m, 331}=\frac{g_{X} \beta_{m}}{\sqrt{6 X_{g}}}, \\
& X_{g}=g^{2}+\frac{g_{X}^{2} \beta_{m}^{2}}{6}, \\
& s_{W}=\sin \theta_{W}=\frac{g_{1}}{\sqrt{g^{2}+g_{1}^{2}}}, \\
& c_{W}=\cos \theta_{W}=\frac{g}{\sqrt{g^{2}+g_{1}^{2}}}, \quad g_{1}=\frac{g g_{X}}{\sqrt{6 X_{g}}}, \\
& g_{X}^{2}=\frac{6 g^{2} s_{W}^{2}}{1-\left(1+\beta_{m}^{2}\right) s_{W}^{2}} .
\end{aligned}
$$

If we choose $h_{1}=+1$ for model $M_{1}$, as in Ref. [14], then we agree with Ref. [10]. For $M_{2}$, we agree with Ref. [12] where the convention $h_{2}=+1$ is used.

The mass matrix in the basis of $\left(A_{\mu}, Z_{\mu}, Z_{\mu}^{\prime}\right)$ for both models is

$M_{A Z Z^{\prime}}^{2}=\left(\begin{array}{ccc}0 & 0 & 0 \\ 0 & m_{22}^{2} & m_{23}^{2} \\ 0 & m_{23}^{2} & m_{33}^{2}\end{array}\right)$,

with

$$
\begin{aligned}
m_{22}^{2}= & \frac{m_{W}^{2}}{c_{W}^{2}}, \quad m_{23}^{2}=-\frac{h_{m} g_{X} m_{W}^{2}}{3 \sqrt{2} g s_{W}}\left(a_{m}+\sqrt{3} \beta_{m} t_{W}^{2}\right), \\
m_{33}^{2}= & \frac{X_{g} v_{m, 3}^{2}}{3} \\
& +\frac{g_{X}^{2} m_{W}^{2}}{18 g^{2} t_{W}^{2}}\left(1+3 t_{W}^{4} \beta_{m}^{2}+2 \sqrt{3} t_{W}^{2} \beta_{m} a_{m}\right),
\end{aligned}
$$

where

$a_{m}=\frac{v_{m, 1}^{2}-v_{m, 2}^{2}}{v_{m, 1}^{2}+v_{m, 2}^{2}}$.

Because of Eq. (21), we have

$a_{1}=-a_{2}$

confirming what we wrote in the Sect. 1.

The two eigenvalues read

$$
\begin{aligned}
& m_{Z_{1}}^{2}=\frac{m_{22}^{2}+m_{33}^{2}-\sqrt{\Delta}}{2}, \\
& m_{Z_{2}}^{2}=\frac{m_{22}^{2}+m_{33}^{2}+\sqrt{\Delta}}{2}, \\
& \Delta=\left(m_{22}^{2}-m_{33}^{2}\right)^{2}+4 m_{23}^{4} .
\end{aligned}
$$

We now introduce the $Z-Z^{\prime}$ mixing discussed in the Sect. 1

$$
\left(\begin{array}{c}
Z_{1}^{\mu} \\
Z_{2}^{\mu}
\end{array}\right)=\left(\begin{array}{cc}
c_{m, \xi} & s_{m, \xi} \\
-s_{m, \xi} & c_{m, \xi}
\end{array}\right)\left(\begin{array}{c}
Z^{\mu} \\
Z^{\prime \mu}
\end{array}\right),
$$

with

$$
\begin{gathered}
s_{m, \xi}=-\frac{m_{23}^{2}}{\sqrt{\left(m_{Z_{2}}^{2}-m_{22}^{2}\right)^{2}+m_{23}^{4}}}, \\
c_{m, \xi}=\frac{m_{Z_{2}}^{2}-m_{22}^{2}}{\sqrt{\left(m_{Z_{2}}^{2}-m_{22}^{2}\right)^{2}+m_{23}^{4}}} .
\end{gathered}
$$

We note here that the sign of $\tan \xi_{m}=s_{m, \xi} / c_{m, \xi}$ is completely determined from the matrix in Eq. (A9), but not the sign of $s_{m, \xi}$ or $c_{m, \xi}$. Here we choose the $c_{m, \xi}>0$ convention, so that the $Z_{1}$ couplings become identical to the $Z$ couplings in the limit $\xi \rightarrow 0$. In this convention, the sign of $s_{m, \xi}$ is determined as in Eq. (A15).

Comparing $M_{1}$ against $M_{2}$ we see that $m_{22}^{2}$ and $m_{33}^{2}$ are identical but $m_{23}^{2}$ can be different in sign depending on the convention of $h_{m}$. The physical masses $m_{Z_{i}}^{2}$ are therefore the same in both models. For the mixing angles we have

$s_{1,331}=s_{2,331}, \quad c_{1,331}=-c_{2,331}, \quad c_{1, \xi}=c_{2, \xi}$,

while $s_{1, \xi}= \pm s_{2, \xi}$ depending on the sign convention of $h_{m}$. $c_{W}$ and $s_{W}$ are chosen to be the same in both models.

In the case of $v_{m, 3} \gg v_{m, 1}, v_{m, 2}$ we have $m_{33} \gg m_{22}, m_{23}$ and

$$
\begin{aligned}
s_{m, \xi} & \approx-\frac{m_{23}^{2}}{m_{33}^{2}} \\
& =\frac{h_{m} c_{W}^{2}}{3 \sqrt{1-\left(1+\beta_{m}^{2}\right) s_{W}^{2}}}\left(3 \beta_{m} t_{W}^{2}+\sqrt{3} a_{m}\right) \frac{m_{22}^{2}}{m_{33}^{2}} .
\end{aligned}
$$

This result agrees with Refs. [10,12] if we choose $h_{1}=h_{2}=$ +1 .

To calculate neutral currents couplings to the leptons we need the diagonal entries of the covariant derivative. Writing $D_{\mu}^{\text {diag }}=\partial_{\mu}+\hat{D}_{\mu}$, in the original basis $\left(X_{\mu}, W_{\mu}^{8}, W_{\mu}^{3}\right)$ we have

$$
\begin{aligned}
& \hat{D}_{m, \mu}^{\text {lepton,L }}=-i \operatorname{diag}\left(\frac{g s_{m}}{2} W_{\mu}^{3}+\frac{g s_{m}}{2 \sqrt{3}} W_{\mu}^{8}\right. \\
& +\frac{g_{X}}{\sqrt{6}}\left(-\frac{1}{2}-\frac{\beta_{m} s_{m}}{2 \sqrt{3}}\right) X_{\mu}, \\
& -\frac{g s_{m}}{2} W_{\mu}^{3}+\frac{g s_{m}}{2 \sqrt{3}} W_{\mu}^{8}+\frac{g X}{\sqrt{6}}\left(-\frac{1}{2}-\frac{\beta_{m} s_{m}}{2 \sqrt{3}}\right) X_{\mu}, \\
& \left.-\frac{g s_{m}}{\sqrt{3}} W_{\mu}^{8}+\frac{g_{X}}{\sqrt{6}}\left(-\frac{1}{2}-\frac{\beta_{m} s_{m}}{2 \sqrt{3}}\right) X_{\mu}\right), \quad s_{m}=(-1)^{m}, \\
& m=1,2,
\end{aligned}
$$


Table 1 Couplings between the $Z, Z^{\prime}$ gauge bosons and the left-, right-handed Standard Model's leptons in the models $M_{1}$ and $M_{2}$

\begin{tabular}{llllll}
\hline Model & Lepton & $\Delta_{L}(Z)$ & $\Delta_{R}(Z)$ & $\Delta_{L}\left(Z^{\prime}\right)$ & $\Delta_{R}\left(Z^{\prime}\right)$ \\
\hline$M_{1}$ & $v$ & $\frac{g}{2 c_{W}}$ & 0 & $\frac{h_{1} g}{2 c_{W}} \frac{1-\left(1+\sqrt{3} \beta_{1}\right) s_{W}^{2}}{\sqrt{3} \sqrt{1-\left(1+\beta_{1}^{2}\right) s_{W}^{2}}}$ & 0 \\
$M_{2}$ & $v$ & $\frac{g}{2 c_{W}}$ & 0 & $-\frac{h_{2} g}{2 c_{W}} \frac{1-\left(1-\sqrt{3} \beta_{2}\right) s_{W}^{2}}{\sqrt{3} \sqrt{1-\left(1+\beta_{2}^{2}\right) s_{W}^{2}}}$ & 0 \\
$M_{1}$ & $e$ & $\frac{g}{2 c_{W}}\left(2 s_{W}^{2}-1\right)$ & $\frac{g}{c_{W}} s_{W}^{2}$ & $\frac{h_{1} g}{2 c_{W}} \frac{1-\left(1+\sqrt{3} \beta_{1}\right) s_{W}^{2}}{\sqrt{3} \sqrt{1-\left(1+\beta_{1}^{2}\right) s_{W}^{2}}}$ & $\frac{h_{1} g}{c_{W}} \frac{-\beta_{1} s_{W}^{2}}{\sqrt{1-\left(1+\beta_{1}^{2}\right) s_{W}^{2}}}$ \\
$M_{2}$ & $e$ & $\frac{g}{2 c_{W}}\left(2 s_{W}^{2}-1\right)$ & $\frac{g}{c_{W}} s_{W}^{2}$ & $-\frac{h_{2} g}{2 c_{W}} \frac{1-\left(1-\sqrt{3} \beta_{2}\right) s_{W}^{2}}{\sqrt{3} \sqrt{1-\left(1+\beta_{2}^{2}\right) s_{W}^{2}}}$ & $-\frac{h_{2} g}{c_{W}} \frac{\beta_{2} s_{W}^{2}}{\sqrt{1-\left(1+\beta_{2}^{2}\right) s_{W}^{2}}}$ \\
\hline
\end{tabular}

and we have used $X_{m}=-1 / 2-\beta_{m} s_{m} /(2 \sqrt{3})$ for lefthanded lepton multiplets.

As in Ref. [10], we define the couplings between the $Z_{1}$ and $Z_{2}$ bosons to the fermions as follows

$\Delta_{m, k}^{f f}\left(Z_{1}\right)=c_{m, \xi} \Delta_{m, k}^{f f}(Z)+s_{m, \xi} \Delta_{m, k}^{f f}\left(Z^{\prime}\right)$,

$\Delta_{m, k}^{f f}\left(Z_{2}\right)=-s_{m, \xi} \Delta_{m, k}^{f f}(Z)+c_{m, \xi} \Delta_{m, k}^{f f}\left(Z^{\prime}\right)$,

where $k=L, R, A, V$ with $\Delta_{V}=\Delta_{L}+\Delta_{R}, \Delta_{A}=\Delta_{R}-$ $\Delta_{L}$, with the following convention

$$
\begin{aligned}
\mathcal{L}_{f}\left(Z, Z^{\prime}\right)= & \bar{f} \gamma^{\mu}\left[\Delta_{L}^{f f}(Z) P_{L}+\Delta_{R}^{f f}(Z) P_{R}\right] f Z_{\mu} \\
& +\bar{f} \gamma^{\mu}\left[\Delta_{L}^{f f}\left(Z^{\prime}\right) P_{L}+\Delta_{R}^{f f}\left(Z^{\prime}\right) P_{R}\right] f Z_{\mu}^{\prime} .
\end{aligned}
$$

Results for these couplings are given in Table 1 for the case of the SM leptons. With the convention $h_{2}=+1$ as in Refs. [12,13], those $l l Z$ and $l l Z^{\prime}$ couplings agree with Refs. $[12,13]$.

In comparison with Ref. [10] we have to choose $h_{1}=$ $h_{2}=+1$ to get the same sign for $s_{m, \xi}$. We agree with them for model $M_{1}$. For model $M_{2}$, which they call $F_{2}$, we can only agree if a minus sign is added to the $l l Z^{\prime}$ couplings.

It is important to note that the physical results such as the $e^{+} e^{-} \rightarrow \mu^{+} \mu^{-}$cross section are independent of $h_{m}$ because it occurs both in the $\Delta_{k}^{l l}\left(Z^{\prime}\right)$ couplings and in $s_{m, \xi}$.
The $Z-Z^{\prime}$ interference terms are independent of $h_{m}$. Using the convention $h_{1}=-h_{2}$, the above results show that the $l l Z$, $l l Z^{\prime}$ couplings, $s_{m, \xi}$, and $c_{m, \xi}$ are the same in both models.

\section{References}

1. M. Singer, J.W.F. Valle, J. Schechter, Phys. Rev. D 22, 738 (1980)

2. J.W.F. Valle, M. Singer, Phys. Rev. D 28, 540 (1983)

3. F. Pisano, V. Pleitez, Phys. Rev. D 46, 410 (1992). arXiv:hep-ph/9206242

4. R. Foot, O.F. Hernandez, F. Pisano, V. Pleitez, Phys. Rev. D 47, 4158 (1993). arXiv:hep-ph/9207264

5. P.H. Frampton, Phys. Rev. Lett. 69, 2889 (1992)

6. R. Foot, H.N. Long, T.A. Tran, Phys. Rev. D 50, 34 (1994). arXiv:hep-ph/9402243

7. L.T. Hue , L.D. Ninh, Mod. Phys. Lett. A 31, 1650062 (2016). arXiv: 1510.00302

8. S. Descotes-Genon, M. Moscati, G. Ricciardi, Phys. Rev. D 1711, 03101 (2017)

9. F. Richard, (2013). arXiv:1312.2467

10. A.J. Buras, F. De Fazio, J. Girrbach-Noe, JHEP 08, 039 (2014). arXiv: 1405.3850

11. A.J. Buras , F. De Fazio, JHEP 03, 010 (2016). arXiv: 1512.02869

12. R. Martinez , F. Ochoa, Phys. Rev. D 90, 015028 (2014). arXiv: 1405.4566

13. A.E. Carcamo Hernandez, R. Martinez, F. Ochoa, Phys. Rev. D 73, 035007 (2006). arXiv:hep-ph/0510421

14. M.V. Carlucci, Quark-Flavour Phenomenology of Models with Extended Gauge Symmetries (Technical University, Munich, 2013). $\mathrm{PhD}$ thesis 